\title{
TEMPERATURE MONITORING AND LOGGING SYSTEM SUITABLE FOR USE IN HOSPITALS, INCORPORATING GSM TEXT MESSAGING
}

\author{
${ }^{1}$ I. G. Saidu, and ${ }^{2}$ M. Momoh and ${ }^{1}$ A. S. Mindaudu \\ ${ }^{1}$ Physics/ Electrical Department Sokoto State Polytechnic, Sokoto \\ ${ }^{2}$ Department of Physics, Usmanu Danfodiyo University, Sokoto. \\ ${ }^{1}$ ismailsaidu258@gmail.com
}

\begin{abstract}
In this paper, a microcontroller-based temperature monitoring and logging system suitable for use in hospitals was designed and constructed. The features include ability to monitor a patient's temperature on a continuous basis while displaying the instant result on a Liquid Crystal Display (LCD) device. The temperature monitored is logged in a memory device, Electrically Erasable Programmable Read Only Memory (EEPROM), located in the system at every 10 minutes interval and can be interfaced with a computer using USB or RS232 UART device. An ATmegal6 AVR is used as the heart of the control and coordination of all the activities of the individual modules. It allowed a doctor at location from the patient to keep track of his patient's condition while attending to other issues.
\end{abstract}

\section{Keywords}

Microcontroller. Patient temperature and data logger.

\section{INTRODUCTION}

A temperature log is a document in which information about temperature is maintained. The log usually consists of temperature readings taken at set intervals over a given period of time. (Smith, 2010). Temperature monitoring has assumed a vital role both in the management of patient's condition and general hospital storage facilities. This is why the American State Department of Health (DOH) and the Joint Commission on Accreditation of Healthcare Organization (JCAHO) require temperature monitoring and logging for all patients' care-related refrigerators, freezers and blanket warmers (iWatch, 2009). Failure to comply can lead to medically significant problems (Zweig, 2005 cited by Presidio, 2009) and costly remediation actions (iWatch, 2009). While manual temperature monitoring logging on paper is still done in some regions of the world, a temperature log is often done digitally (Smith, 2010). There are however some distinct advantages to logging digitally as it allows for automatic logging that reduces the rate of human error (Presidio, 2009) and in streamlining the logging process(Smith, 2010). According to Presidio (2009) "To meet the demands of the tight-margin, competitive market- place, hospitals and other healthcare providers can no longer afford to rely on manual processes and out-dated technology".

Several temperature monitoring and logging systems have been presented in quite a number of publications. The designs exhibit different unique features. For instance, Kapidere et al., (2005)

DOI : $10.5121 /$ ijist.2013.3102 
designed and constructed a microcontroller based human brain hypothermia system intended for cooling and heating brain. Robert et al., (1999) developed a data logging system with two units; a logger and a reader which work with a host computer. They used EEPROM chip to store data and PIC 16C73A as the controller. Singh (2006) designed and developed a microcontroller based temperature and humidity controller for an infant incubator. It monitors and controls the two parameters constantly which are very critical for the normal growth of the new born (premature) babies. Lewis (1995), in a research, developed a system that helps parents monitor the temperature of babies remotely. The system continuously monitors the temperature and sounds an alarm when the temperature exceeds a pre-set threshold.

In similar work a temperature and pressure monitoring system was fitted to the tyre of offhighway trucks so that the behaviour of the tires can be studied. It's made to act when variations exceed the normal operation parameters. In addition it provides statistical information of tyre usage, yield, and lifespan and wears (Olavaria, 2006).

In a work by Raza (2010), a microcontroller based system for data acquisition and logging as needed was designed. The device could be used for monitoring and controlling environment temperature. He employed AT89C51 to send the result of 8 sensors. Qingsheng and Julio (2004) invented a device capable of core body temperature monitoring in heart failure. The heart monitoring system comprised of a temperature sensor for determining a core body temperature of a patient configured to receive data representative of the core body temperature and the bodily activity level. Tiplady (2010) invented a remote critical area monitoring system that allows connection to a web page to obtain video image of some parameters. Other inventions, worth mentioning, include that of Goswani et al. (2009), an embedded system capable of monitoring temperature and light and that of Deepika (2006) a data logging system based on 8051 family and the result stored in EEPROM.

Generally, the above designs offer a better substitute to the manual monitoring and logging of temperature systems, they mostly require separate modules and lack certain features. Moreover, this type of device is lacking in hospitals of the developing countries. This is why this research was initiated with the aim of incorporating other useful features

\section{DESIGN}

The device was constructed as a stand-alone system with the various functional circuits assembled as separate detachable modules linked together by cable buses. Each module performs specific function as directed and instructed by an AVR microcontroller chip of the type ATmega16. The general block diagram of the system is as shown in fig. 1. 


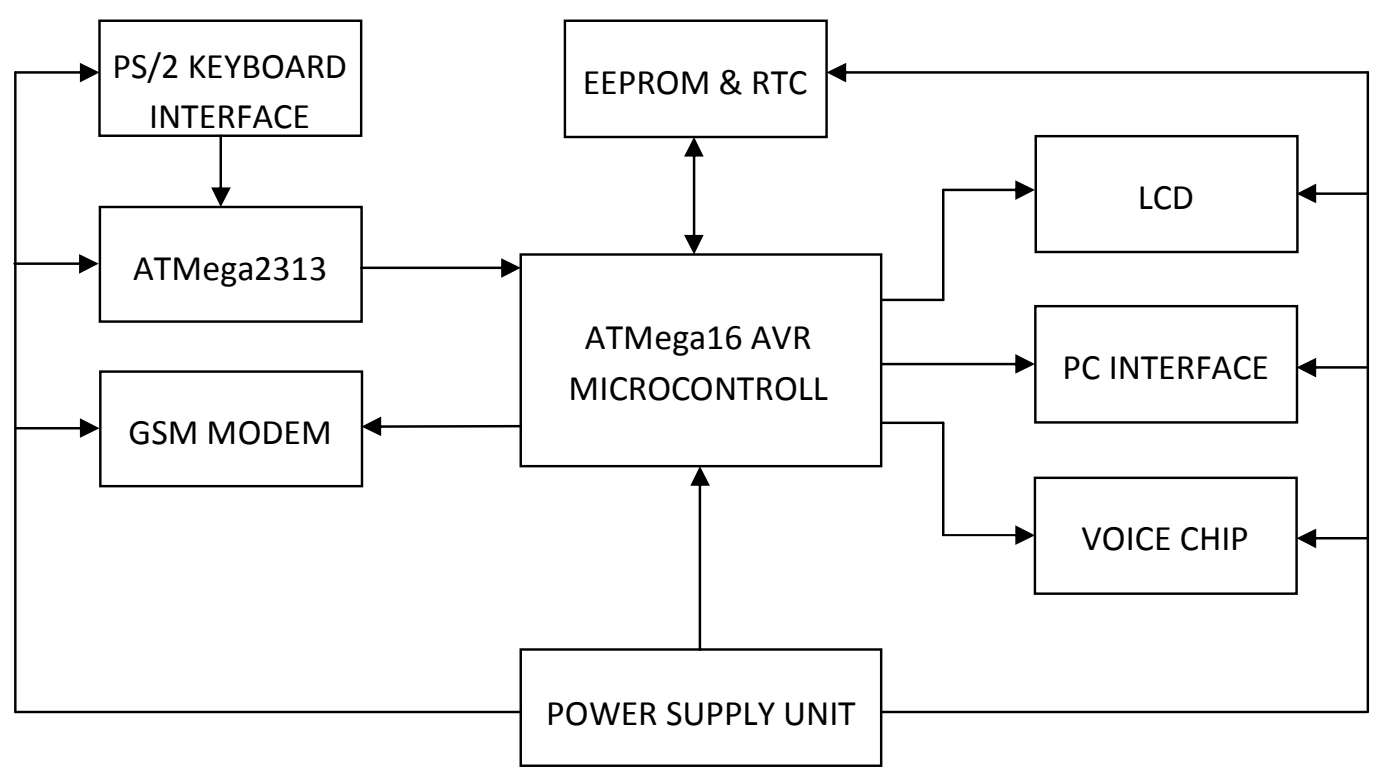

Fig. 1 Block Diagram of the System

\section{The power supply unit design}

The PSU for the system should be capable of providing the required supply voltage and current to all the various sub-units in the system. Except for the GSM modem included, which is responsible for sending short messages when required, all other sub-units require a dc supply of $5 \mathrm{~V}$ with a current drain in the region of 2 Amperes (AVR ATmega16 Data book, 2001). The GSM modem requires a dc supply of 4.2V (GSM Modem data book, 2005). The PSU is based on a full wave bridge rectifier including suitable smoothing filters and an appropriately rated transformer. Three regulators were employed for each sub-unit to ensure that the required regulated voltage was obtained.

The circuit diagram of the PSU is in Fig 2 employing a step down transformer, a bridge rectifier, a smoothing circuit and three separate regulators. Two separate sources of $5 \mathrm{~V}$ regulated outputs were provided in order not to overload any of the regulators. One of the $5 \mathrm{~V}$ supplies was reserved for the relay circuit as it draws large current. 


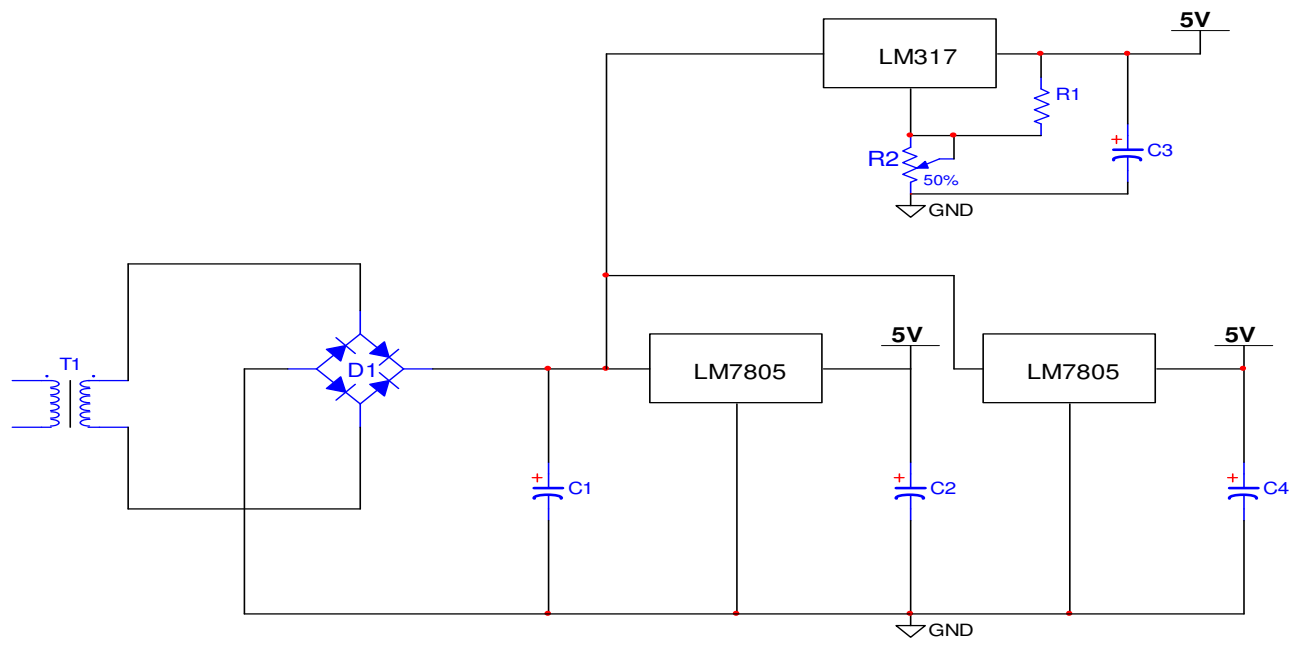

Fig 2: PSU circuit diagram

\section{The Main Micro (At Mega 16) Circuit Design}

The heart of the system is the AVR microcontroller. The 40pin dip is used to control the activities of all other sections. ATmega16 AVR was selected due to its good features of being cheap and readily available in the market. The pin assignment of the microcontroller is as shown in fig 3

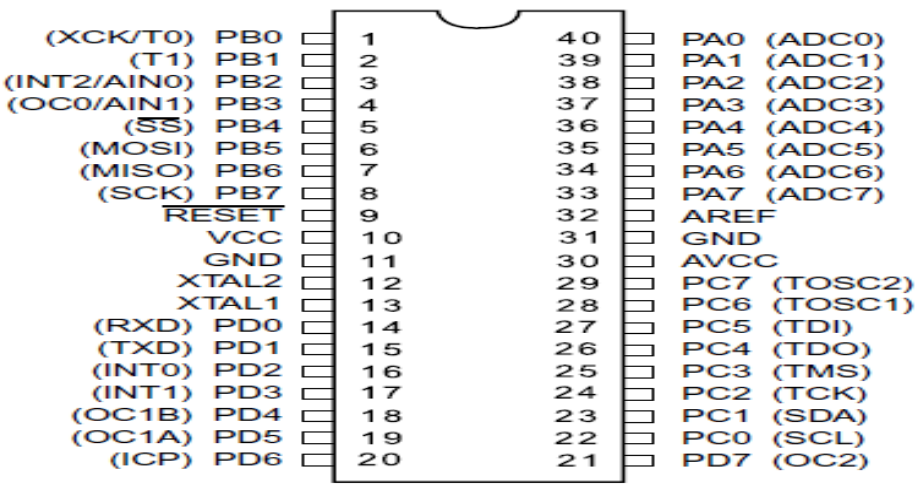

Fig.3 Pinouts of ATmega16 (ATmega16 data book, 2001)

The microcontroller datasheet recommends two separate voltage lines for the IC, one for the Digital signal circuit and the other for analogue signal. The analogue voltage is for the ADC circuit and it is suggested that the value should not differ from the $\mathrm{V}_{\mathrm{cc}}$ by $\pm \mathrm{O}$. $3 \mathrm{~V}$.

In this circuit, the $\mathrm{V}_{\mathrm{cc}}$ voltage of $5 \mathrm{~V}$ was selected and separately connected to the analogue voltage terminals of the IC. In other to minimise the effect of the digital signal on the accurate conversion of the ADC circuit, an external capacitor of $100 \mathrm{nF}$ was connected between the analogue supply terminals of the chip. This ensures analogue noise cancelling in the circuit.

The Digital voltage supply is between 4.5 and 6.5 volts. For this circuit a $5 \mathrm{~V}$ regulated supply was used as the $\mathrm{V}_{\mathrm{cc}}$ of the circuit. The positive terminal was fed to pin 10 and the ground terminal to pin 11. Pins 12 and 13 serves as the crystal input and output of an inverting amplifier and it was configured as an On-chip oscillator. The connection as recommended (ATmega16 data book, 2001 ) is that the values of the capacitors be in such a manner that $C_{1}=C_{2}$. And that the value 
should be between 12 and $22 \mathrm{pF}$. For these reasons we used 2 numbers of $22 \mathrm{pF}$ capacitors and a crystal with a value of $32.768 \mathrm{kHz}$.

\section{Temperature Sensor And ADC Circuit}

The temperature sensing arrangement used in the system is as designed in (Ismaila and Momoh, 2011), resulting in the circuit shown in Fig. 4. The reference voltage for the ADC and LM35 were chosen to be $5 \mathrm{~V}$.

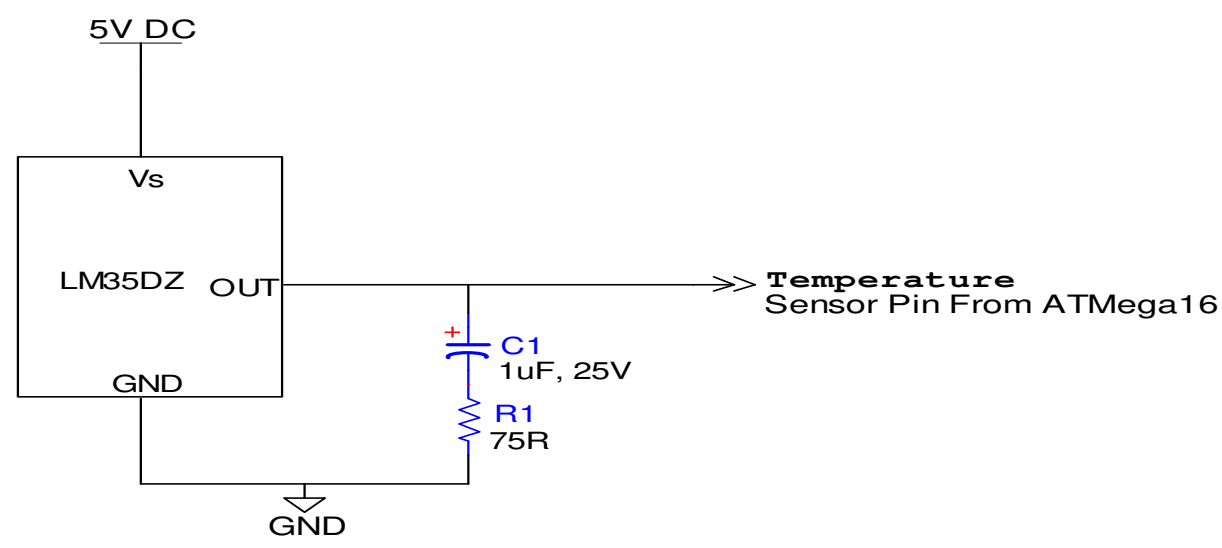

Fig. 4 LM35 Temperature Sensing Circuit (Ismaila and Momoh, 2011)

\section{Keyboard Interface}

In operation the main micro at certain interruption copies data in the EEPROM and send it to the LCD, the PC or MODEM for transmission. To do this, the addresses in the EEPROM are used to hold patient's data such as name, age, sex etc. These data can be changed when desired. To change these data a form of external human interface is required. The design used a standard PC AT keyboard as interface to transfer data to the main micro.

The keyboard is powered from the $+5 \mathrm{~V}$ dc supply which is available through the keyboard interface. A PS/2 connector is used to connect the keyboard to the 20 pin microcontroller.

The LEDs connected to the ports of 18 to 12 serves as the indicator of the status of the outputs of port D. Resistors R1 through R7 are current limiting resistors that sink current from the voltage supply and the can be obtained from the equation:

$\mathrm{R}=\left(\mathrm{V}_{\mathrm{cc}}-\mathrm{V}_{\mathrm{LED}}\right) / \mathrm{I}$

From the data sheet of the LED, the $\mathrm{V}_{\mathrm{LED}}$ is $2.2 \mathrm{~V}$ and $\mathrm{V}_{\mathrm{cc}}=5 \mathrm{~V}$, while $\mathrm{I}=30 \mathrm{~mA}$, so that $\mathrm{R}=110 \Omega$.

A value of $100 \Omega$ was chosen as the nearest value for all the current limiting resistors as same type of LEDs are used as indicators throughout the design.

The final circuit connection of the Atiny 2313 is as shown in fig 5 


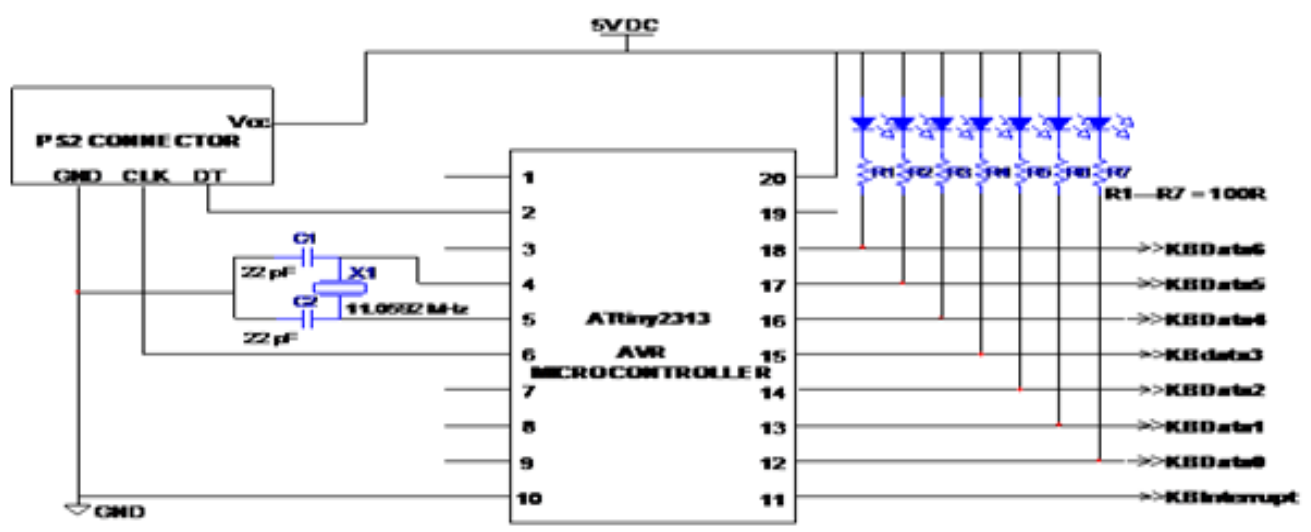

Fig 5 ATiny 2313 complete circuit

During operation, whenever there is a valid converted scan code signal from the keyboard IC the data is received serially by the micro. It uses the program stored in the program memory of the micro to convert it to ASCII value. The ASCII value is transmitted to the main micro by interrupting the main micro's activity through an enabling signal from pin 11 of the ATiny 2313. The main micro then reads the output data at the output of the ATiny2313. The data is first converted to parallel format using the internal UART located in the ATiny 2313 microcontroller. Port A of the AVR ATmega16 was selected and used to receive the converted ASCII code value from the ATiny 2313 microcontroller. Pin 16 (INT0) serves as the external interrupt of the chip which can be triggered by either a falling edge or rising edge signal.

\section{PC Interface Circuit}

The logged data in the EEPROM can be assessed by a PC through a PC interfaced to the main micro. Because of voltage level differences of the devices, a MAX 232 was used to provide voltage matching of the different types of logic used in the microcontroller and those in the P C. The Max 232 RS232 is a 16 pin dip IC that is used to convert CMOS voltages to TTL voltages. As recommended in the datasheet of MAX232, () application note, the circuit shown in Fig 6 was adopted.

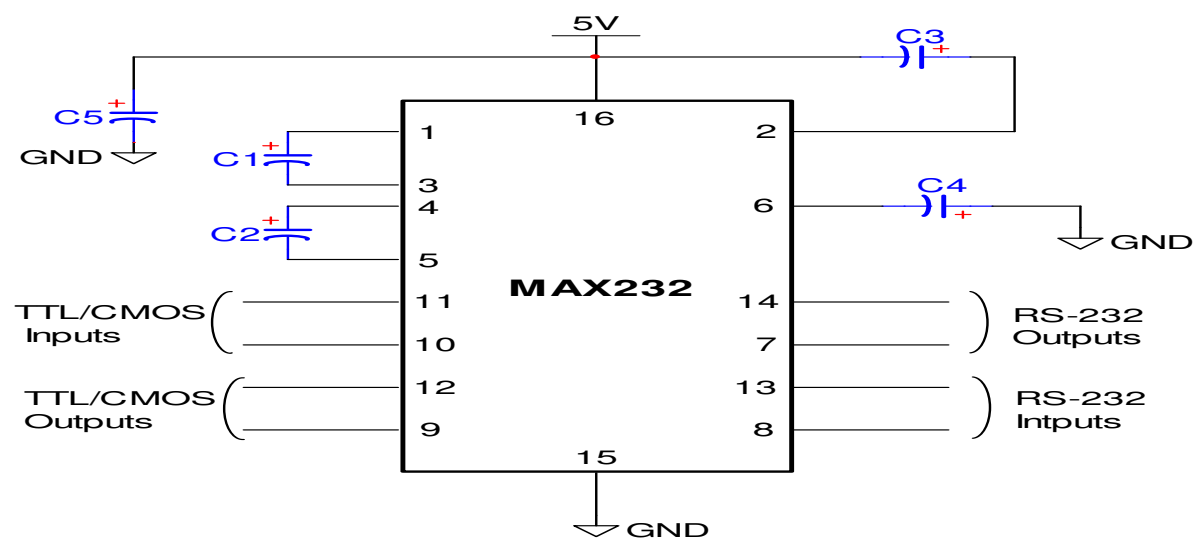

Fig 6 MAX 232 complete circuit 
The GSM and PC interface were made to share the same line. For this reason a manual switch was provided. Whenever the GSM is being used, the PC interface is disconnected and the bus is switched over to the GSM modern. Similarly when there is need to load the logged data to the PC, the GSM modern is disabled and the common bus is switched over to serve the PC request

\section{GSM Circuit Design}

Fig 8 showed the GSM modem circuit and as recommended in the data sheet of the device the supply voltage is $4.2 \mathrm{~V}$. A stabilised dc voltage source of $4.2 \mathrm{~V}$ was used to supply the modem. It is also recommended that two pull-up resistors of values $4 \mathrm{~K} 7$ be used to connect the two terminals of the EN and RX to the positive supply line. Capacitors $\mathrm{C} 1$ and $\mathrm{C} 2$ are decoupling capacitors with recommended values of $10 \mathrm{uF}$ each (GSM Modem data book, 2005). Capacitor C2 is to prevent the bounce of the switch from offsetting the working condition of the device.

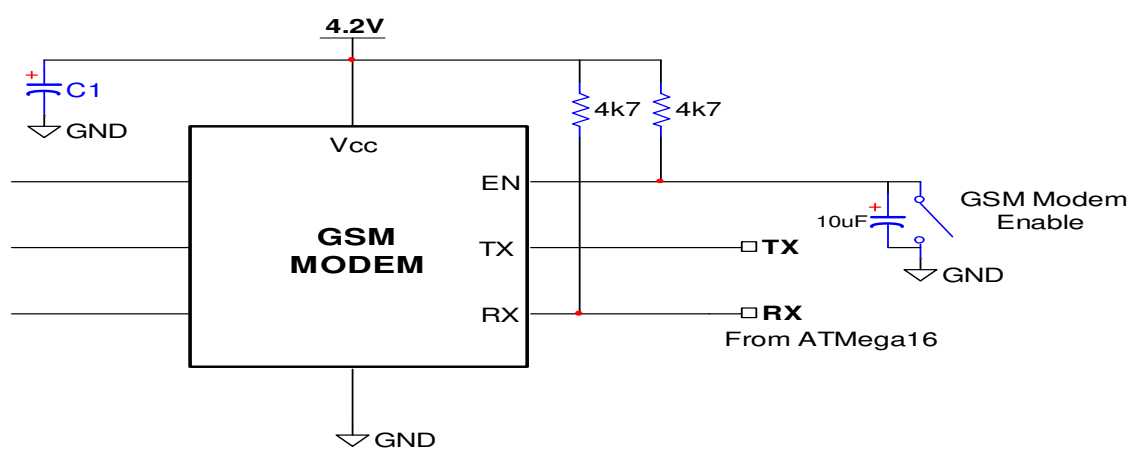

Fig 7 GSM modem circuit

\section{Microcontroller as Two-Wire Serial Interface Circuit (EEPROM and RTC)}

The microcontroller was used as the master in a two-wired interface (TWI) communication scheme with the EPROM and RTC as the slaves (Fig. 9). The TWI protocol allows the system designer to interconnect up to 128 different devices using only two bi-directional bus lines, one for clock (SCL) and one for data (SDA).

The microcontroller was configured using software to initiate and terminate a transmission. It also generates the SCL for the operation of the system. The supply voltage is recommended to be between $0.7 \mathrm{~V}_{\mathrm{cc}}$ and $\mathrm{V}_{\mathrm{cc}}+0.5$ (ATmega16)

But $\mathrm{V}_{\mathrm{cc}}=5 \mathrm{~V}$, then

$\mathrm{V}_{\text {min }}=0.7 \times 5=3.5 \mathrm{~V}$

$\mathrm{V}_{\text {max }}=5+0.5=5.5 \mathrm{~V}$

It was decided that the supply voltage be $5 \mathrm{~V}$ since this is already available from the main supply. For the input low voltage the data book recommends a voltage of between $-0.5 \mathrm{~V}$ and $0.3 \mathrm{~V}_{\mathrm{cc}}$ This gives a range of -0.5 to $0.3 \times 5$ or -0.5 to $1.5 \mathrm{~V}$

The ground of $\mathrm{V}_{\mathrm{cc}}$ was used as the low voltage. 


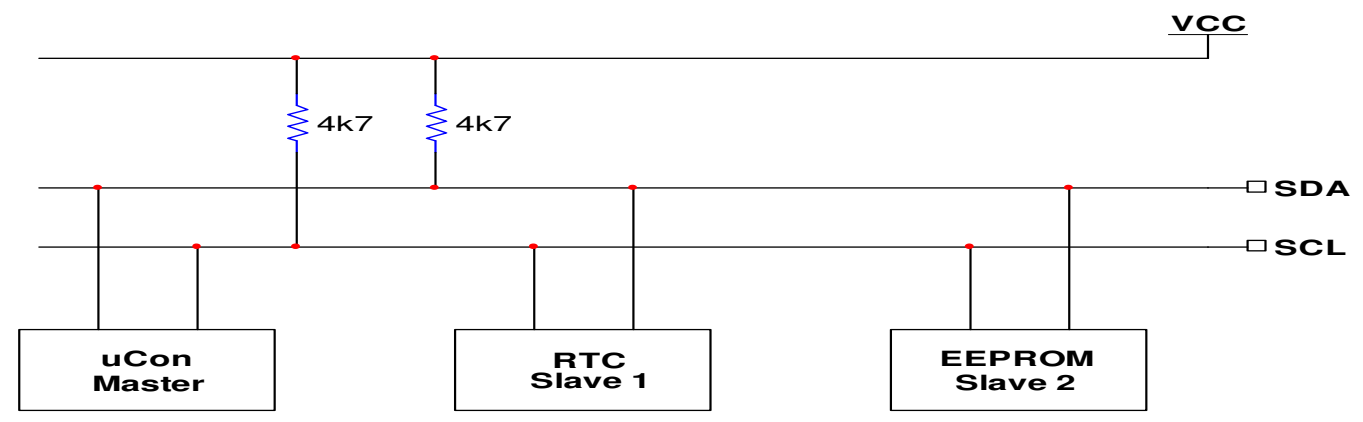

Fig 8 the two wire interface

The datasheet recommends the frequency of operation to be between 0 to $400 \mathrm{KHz}$. The frequency selected was $200 \mathrm{KHz}$.

For this frequency it is recommended that the value of pull-up resistor should be calculated using $\mathrm{R}_{\mathrm{p}(\min )}=\left(\mathrm{V}_{\mathrm{cc}}-0.4 \mathrm{~V}\right) /$ (sink current)

where $\mathrm{R}_{\mathrm{p}(\min )}=$ minimum value of pull up resistor

substituting $\mathrm{V}_{\mathrm{cc}}=$ supply voltage $=5 \mathrm{~V}, \mathrm{~V}=$ voltage supply of the TWI line $=\mathrm{V}_{\mathrm{cc}}$ and sink current $=3 \mathrm{~mA}(\mathrm{GSM}$ Modem data book, 2005). Then

$\mathrm{R}_{\mathrm{p}(\min )}=(5-0.4 \times 5) /(3 \mathrm{~mA})$

$(3 \mathrm{~mA})=$ is the sink current

(GSM Modem data book, 2005).

$\mathrm{R}_{\mathrm{p}(\min )}=1 \mathrm{~K} \Omega$

and $\mathrm{R}_{\mathrm{pmax}}$ is given as

$\mathrm{R}_{\mathrm{p}(\max )}=\mathbf{1 0 0 0} / \mathbf{C}_{\mathrm{b}} \quad$ (GSM Modem data book, 2005).

where $\mathrm{C}_{\mathrm{b}}$ is capacitance of bus line assumed to be $200 \mathrm{pF}$, so that

$\mathrm{R}_{\mathrm{p}(\max )}=5 \mathrm{k} \Omega$

The pull up resistor is therefore to be between $1 \mathrm{~K}$ and $5 \mathrm{~K}$.

We therefore chose a standard value of $4.7 \mathrm{~K}$ as the pull up resistors for each of the two lines. We also note that this result applies to the choice of other pull-up resistors in the entire design.

Whenever there is a request for writing or reading to the EEPROM, either to write or read, the request is inputted through the keyboard interface circuit to the main micro. The main micro then places the address of the EEPROM followed by either an instruction to read or write from/to the EEPROM. The data retrieved may be sent to the LCD for display, sent to the GSM modem for onward transmission to a phone address in another location or sent to a PC interfaced through the main microcontroller.

The external EEPROM was used to log the temperature data of the patient at 10 minutes intervals. A 24C08B EEPROM was selected because of its good features and availability. It has 2 wire serial interface bus, $1^{2} \mathrm{c}$ compatibility (EEPROM data book, 1998). The microcontroller serves as the master while the AT2408 EEPROM and the RTC serves as slaves. The master was configured in such a way that the main micro generates the serial clock and controls accessibility to the bus.

As recommended in the data sheet, pins 1 to 4 and 7 should be grounded. The chip requires a supply of between $+4.5 \mathrm{~V}$ to $5.5 \mathrm{~V}$ power supply. A d.c power of $5 \mathrm{~V}$ was therefore selected to power the device. The EEPROM is organized into 8 pages or blocks of 256 bytes each. It is capable of retaining data for 200 years. It also has a page-write buffer for up to 16 bytes 
(EEPROM data book, 1978). Only a page of the 8 pages was used and is capable of logging data at 10 minutes interval for 24 hours. After 24 hours any further logging will over-write the previous $\log$ in a format of first in first out (erase). The other 7 pages were left unused due to the fact that additional programming memory in the main micro would be required to address and retrieve data from the extra pages. Unfortunately the program memory is very limited and filled up. Using the other pages would have allowed for logging of data for 8 days at 10 minutes interval. Adjustment of the temperature logging interval to say 30 minutes would lead to almost a month's logging of data from the patient. An interface is however provided to enable the data available in the EEPROM to be accessed by a PC.

\section{Real Time Clock Circuit (RTC)}

A real time clock is use to keep track of the current time for some operations of the main micro. It is used to maintain accurate time even when there is no power supply to the system.

The main micro has provision for timing but a separate RTC (Fig 9) is used because of its lower power consumption and it frees the main micro for time critical tasks and it is (RTC, data sheet, ) accurate.

A lithium battery was used to provide a source of alternative power supply to the RTC.

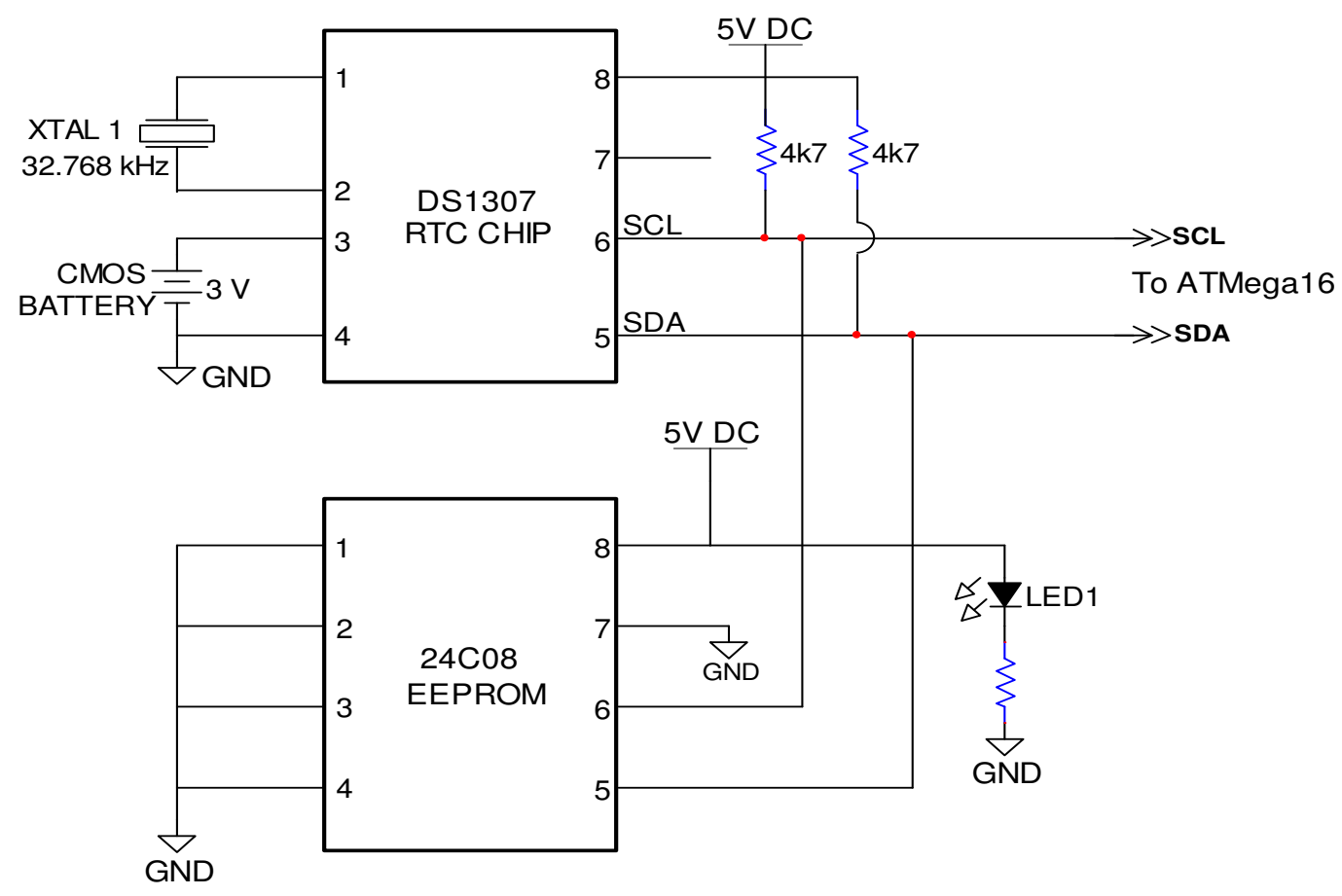

Fig 9 The RTC and EEPROM complete circuit diagram

\section{C D Circuit Design}

Based on the recommendations in the data book of the AVR microcontroller and that of the $16 \times 2$ LCD, (Ismaila and Momoh, 2011) came up with an LCD circuit shown in Fig. 10 which was found suitable for our purpose, was used. 


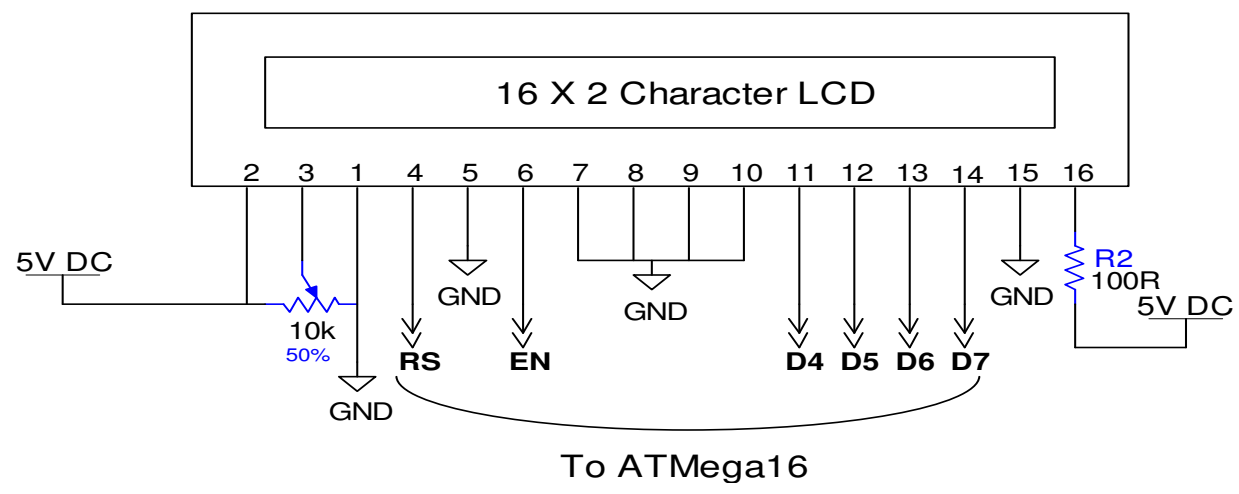

Fig 10 The LCD circuit (Ismaila and Momoh, 2011)

The eventual circuit diagram of the main microcontroller is as shown in fig 11 below. It shows the connections of the various sections to the ATmega16 microcontroller

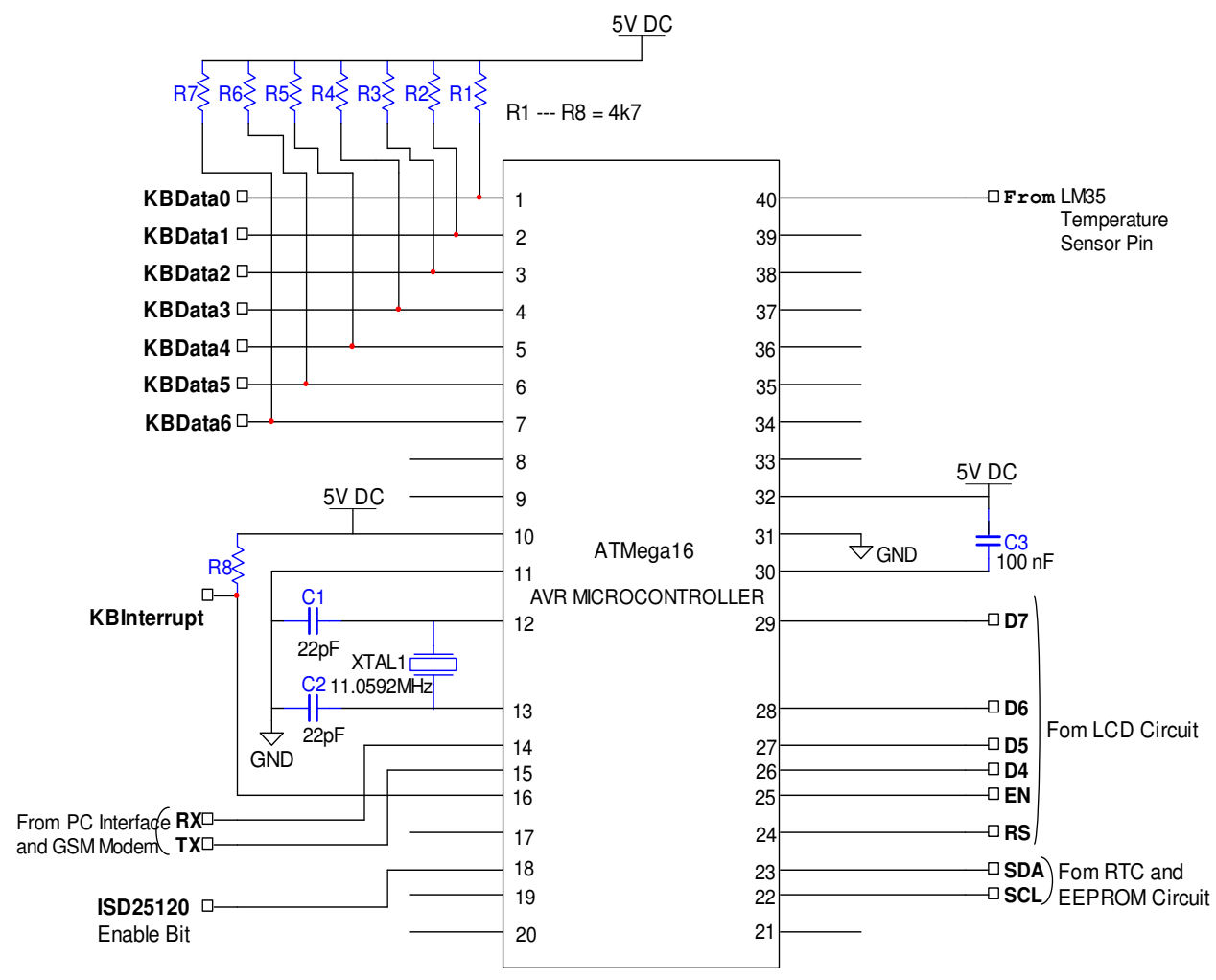

Fig 11 Complete circuit diagram of ATmega16

\section{CONSTRUCTION AND TESTING}

After the design of the circuit each module was tested on bread board and then constructed on separate boards for detachability. The modules were connected together using bus cables and cased on a wooden case. The completed work was subjected to further tests to ascertain the design claim. Plate 1 showed the arrangement for the circuit tests. 


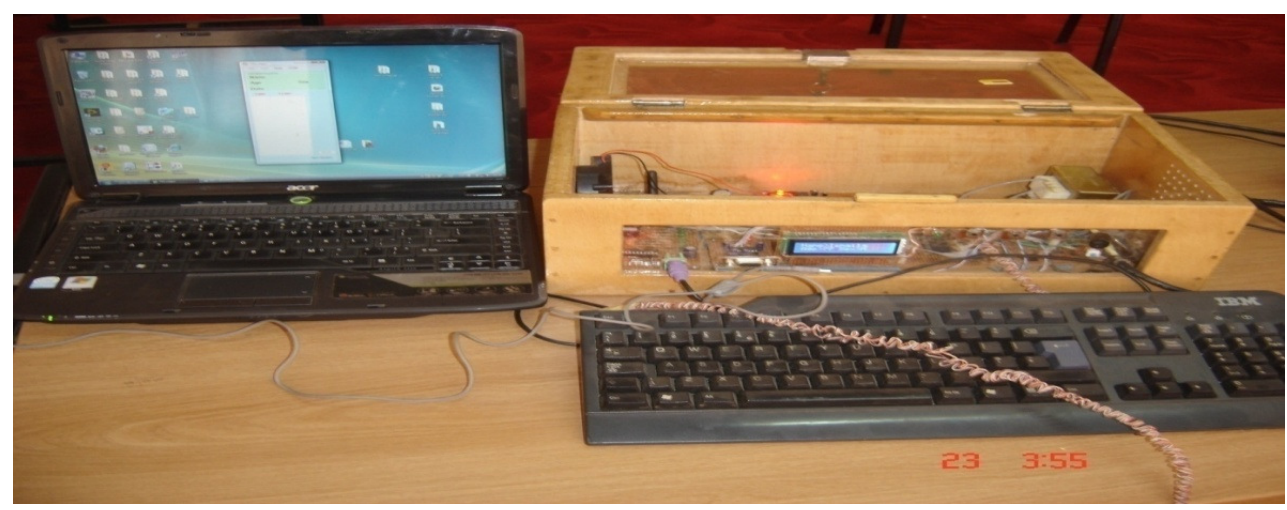

Plate 1 Arrangement for circuit test

\section{Power Supply Test}

The power supply was connected and the voltages at the output of the three regulators were measured using a digital meter. The results got were tabulated.

\section{Thermometer Accuracy Test}

To test for the accuracy of the systems temperature measurement a digital thermometer type (ST-9269 MULTI-STEM THERMOMETER) was obtained and used to conduct an experiment to compare the temperature of the project's thermometer.

A water jug was obtained and half- filled with water. A water heater was inserted into the water while the two thermometers were hung just above the covered water to measure the temperature of the steam just above the water. The temperature of the water was raised gradually by switching on the water heater. The temperature of the enclosure was measured using the two thermometers at intervals and the results tabulated.'

\section{ATiny2313 Conversion and Keyboard Test}

To test to see if the ATiny 2313 was converting the scan code to ASSCI form the keyboard was connected to the circuit through the PS/2. The keys configured were pressed individually and the corresponding response of the LEDs noted. A sample of the ATiny 2313 conversion of keyboard output was then tabulated. The function keys were pressed one after the other and the response as displayed on the LCD monitored.

\section{The RTC accuracy Test}

The accuracy of the RTC time constructed was tested by comparing its time with that of a digital clock. The two times were set at 9.00AM the first day. There times after 48hours were noted and the results recorded.

\section{The GSM Massage Test}

The keyboard was used to set the pre-set temperature arbitrarily, to $43^{0} \mathrm{C}$. A hot soldering iron was carefully placed close to the LM35 sensor to raise its temperature above the pre-set 
temperature. A handset was used to monitor the text massage from the system. The procedure was repeated for several other pre-set temperatures.

\section{Temperature Logging and Computer Interface Test}

The next test conducted was to log a human temperature for a period of six hours. The LM35 sensor was attached to the armpit of a person and used to monitor the temperature for six hours. The temperature logged was interfaced to a computer as shown in the setup of plate 1 to copy the logged temperatures.

\section{RESULTS}

\section{Power Supply Test Result}

The results of the output of the three regulators are as tabulated in Table 1

Table 1 Result of output voltages

\begin{tabular}{|l|l|}
\hline Output of $1^{\text {st }}$ LM7805 & $5 \mathrm{~V}$ \\
\hline Output of $2^{\text {nd }}$ LM7805 & $5 \mathrm{~V}$ \\
\hline Output of LM317 & $4.2 \mathrm{~V}$ \\
\hline
\end{tabular}

Thermometer Accuracy Test Result

The results of the comparism between the temperature measured by the project thermometer and that of the digital thermometer are plotted in Fig 12 below.

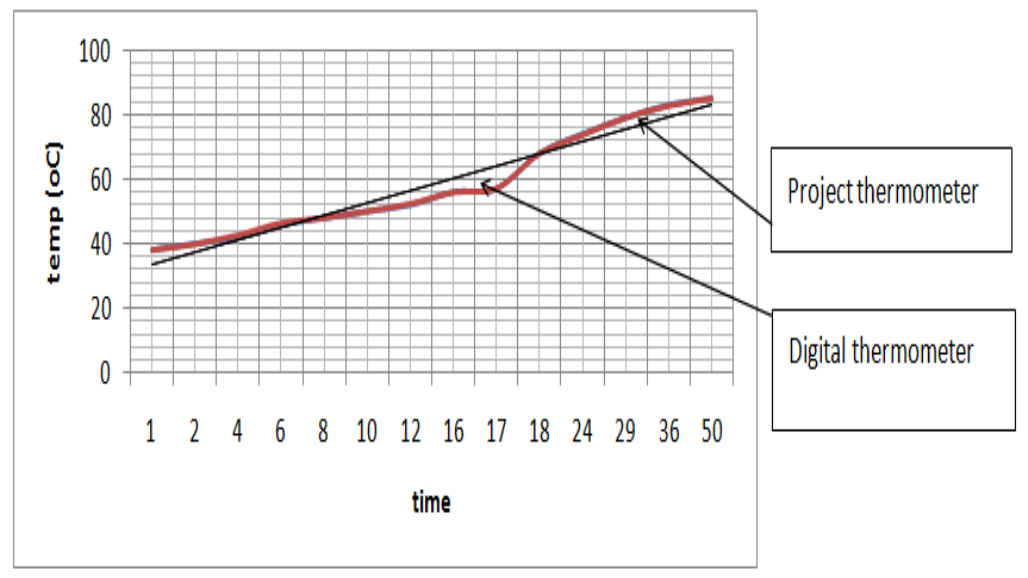

Fig 12 A graph of the measured temperatures against time.

\section{ATiny2313 Conversion and Keyboard Test Result}

A sample of the result of the test on the conversion of scan code to ASCII by ATiny2313 microcontroller is as tabulated in Table 2 
International Journal of Information Sciences and Techniques (IJIST) Vol.3, No.1, January 2013

Table 2 Result of the ATiny2313 Conversion and keyboard test

\begin{tabular}{|l|l|l|l|l|l|l|l|}
\hline KEY & L7 & L6 & L5 & L4 & L3 & L2 & L1 \\
\hline ESC & 0 & 0 & 1 & 0 & 0 & 0 & 0 \\
\hline F1 & 0 & 0 & 0 & 0 & 0 & 0 & 1 \\
\hline F2 & 0 & 0 & 0 & 0 & 0 & 1 & 0 \\
\hline F3 & 0 & 0 & 0 & 0 & 0 & 1 & 1 \\
\hline F4 & 0 & 0 & 0 & 0 & 1 & 0 & 0 \\
\hline F5 & 0 & 0 & 0 & 0 & 1 & 0 & 1 \\
\hline F6 & 0 & 0 & 0 & 0 & 1 & 1 & 0 \\
\hline F7 & 0 & 0 & 0 & 0 & 1 & 1 & 1 \\
\hline F8 & 0 & 0 & 0 & 1 & 0 & 0 & 0 \\
\hline F9 & 0 & 0 & 0 & 1 & 0 & 0 & 1 \\
\hline F10 & 0 & 0 & 0 & 1 & 0 & 1 & 0 \\
\hline F11 & 0 & 0 & 0 & 1 & 0 & 1 & 1 \\
\hline F12 & 0 & 0 & 0 & 1 & 1 & 0 & 0 \\
\hline
\end{tabular}

\section{Result of the RTC Test}

The result of Table 3 is the summary of the test on how accurate the RTC is able to keep time.

Table 3 RTC accuracy test

\begin{tabular}{|l|l|l|}
\hline Date & RTC $(\mathrm{T} 1)$ time & Clock $(\mathrm{T} 2)$ time \\
\hline $10 / 4 / 2011$ & $9.00 \mathrm{am}$ & $9.00 \mathrm{am}$ \\
\hline $12 / 6 / 2011$ & $9.00 \mathrm{am}$ & $9.03 \mathrm{am}$ \\
\hline
\end{tabular}

\section{The Result of GSM Messaging}

It was noted that every time the temperature of the sensor, as displayed on the LCD, exceeds the pre-set temperature by $1^{0} \mathrm{C}$ a text massage was received on the monitoring handset. The result of the text received from the GSM modem is tabulated in Table 4.

Table 4 GSM message

Preset temp $\left(\mathrm{T} 1\right.$ in $\left.{ }^{\circ} \mathrm{C}\right) \quad$ sent temp $\left(\mathrm{T} 2\right.$ in $\left.{ }^{\circ} \mathrm{C}\right)$

43

36

40

45

48

50
44

37

41

46

49

51

\section{Temperature Logging and Computer Interface Test Result}

The temperature logged for 24 hours as logged by the EEPROM and copied to the computer is tabulated in Table 5 . 
Table 5 Result of the temperature logged data for 24 hours.

\begin{tabular}{|l|l|l|l|l|l|l|l|}
\hline Time & Temp. $\left({ }^{0} \mathrm{C}\right)$ & Time & Temp. $\left({ }^{0} \mathrm{C}\right)$ & Time & Temp. $\left({ }^{0} \mathrm{C}\right)$ & Time & Temp. $\left({ }^{0} \mathrm{C}\right)$ \\
\hline 12.00 & 36 & 15.00 & 36 & 18.00 & 35 & 21.00 & 35 \\
\hline 12.10 & 36 & 15.10 & 36 & 18.10 & 35 & 21.10 & 35 \\
\hline 12.20 & 36 & 15.20 & 37 & 18.20 & 35 & 21.20 & 35 \\
\hline 12.30 & 36 & 15.30 & 37 & 18.30 & 35 & 21.30 & 35 \\
\hline 12.40 & 36 & 15.40 & 37 & 18.40 & 35 & 21.40 & 35 \\
\hline 12.50 & 36 & 15.50 & 36 & 18.50 & 35 & 21.50 & 35 \\
\hline 13.00 & 36 & 16.00 & 36 & 19.00 & 36 & 22.00 & 35 \\
\hline 13.10 & 36 & 16.10 & 36 & 19.10 & 36 & 22.10 & 35 \\
\hline 13.20 & 36 & 16.20 & 36 & 19.20 & 36 & 22.20 & 35 \\
\hline 13.30 & 36 & 16.30 & 36 & 19.30 & 36 & 22.30 & 35 \\
\hline 13.40 & 36 & 16.40 & 36 & 19.40 & 35 & 22.40 & 35 \\
\hline 13.50 & 36 & 16.50 & 36 & 19.50 & 35 & 22.50 & 35 \\
\hline 14.00 & 36 & 17.00 & 36 & 20.00 & 35 & 23.00 & 35 \\
\hline 14.10 & 36 & 17.10 & 36 & 20.10 & 35 & 23.10 & 35 \\
\hline 14.20 & 36 & 17.20 & 36 & 20.20 & 35 & 23.20 & 35 \\
\hline 14.30 & 36 & 17.30 & 36 & 20.30 & 35 & 23.30 & 35 \\
\hline 14.40 & 36 & 17.40 & 36 & 20.40 & 35 & 23.40 & 35 \\
\hline 14.50 & 36 & 17.50 & 36 & 20.50 & 35 & 23.50 & 35 \\
\hline
\end{tabular}

\section{DISCUSSION}

The power supply test results are exactly as anticipated (Table 1). When switched on for about one hour the voltage regulators were noted to heat up slightly. For this reason a small fan was attached to the project to blow air over the components to prolong their lifespan.

The results obtained as plotted in Fig. 12 show that there are small deviations between the two temperatures. The possible reason for slight deference in the measured temperatures is that the resolution of the ADC is 1 . This means that temperatures are rounded up to the nearest whole number during $\mathrm{ADC}$ conversion process. The measured temperature is therefore likely to have error of 0 to \pm 0.5 from the actual value.

The results of ATiny 2313 conversion test as shown in Table 2 show that the output LEDs condition is exactly the ASCII equivalent of the numbers pressed. This shows that the key values are being properly converted in the ATiny 2313 microcontroller. The function keys pressed also gave the correct response on the LCD. This is an indication that the function keys are well configured.

The result of the test on the accuracy of the RTC (Table 3) showed that for a period of two days (48 hours) there is an error of 3 minutes which is not too much considering the purpose for which it is designed.

It could be seen from table 4 that whenever the pre-set temperature is exceeded by $1^{0} \mathrm{C}$ a text massage was received on the monitoring handset. It is therefore recommended that the pre-set temperature should be $1^{0} \mathrm{C}$ less than the threshold temperature. In other words if the critical 
patient temperature is $35^{\circ} \mathrm{C}$, then the pre-set temperature should be set at $34^{\circ} \mathrm{C}$. It is also recommended that the MODEM SIM card be charged with sufficient credit to send messages.

The result of Table 5 is an indication that the device is able to log temperature and interface the logged data with a computer as claimed by the design. The analyses of the logged temperature is however not the scope of this paper.

\section{CONCLUSSION}

In this paper, a system has been successfully design that should prove very useful in the field of health care delivery in Nigeria. It is designed to help manage the temperature of a patient that is possibly critically ill in the hospital or to monitor the temperature of other hospital operations such as preservation of drugs, foods and there likes.

The design work is found to meet specification as it is capable of logging temperature on a continuous base and send text massage whenever a pre-set temperature is exceeded.

The major limitation is that the accuracy of the measured value may have error of $\pm 0.5^{\circ} \mathrm{C}$ which may be improved upon by exploring other sensors and improving the ADC resolution.

The LM35 sensor is to be physically attached to the body of the patient to monitor his temperature which has been noted to be inconveniencing. For future development a remote sensor that could monitor the patient's temperature with no physical contact should be considered.

\section{REFERENCES}

[1] ATiny2313 Data Book. (2003, September). AVR Microcontroller. Retrieved April 12, 2011, from ATmel Corporation: http;/www.atmel.com

[2] ATmmega16 Data Book. (2001, November 4). Atmega16. Retrieved May 5, 2011, from ATmel corporation: http;//www.atmel.com

[3] Deepika. (2006). Designing a Microcontroller Based Temperature Data Logger. Patiala, India: Thapar Institute of Engineering and Technology, Deemed University.

[4] DS1307 RTC Data Book. (2001, February 4). Serial Real Time Clock. Retrieved May 4, 2010, from Dalas Semiconductor Corporation Web Site: http;//www.dalassemiconductor.com

[5] EEPROM. (1998). 24C08B/16B Serial EEPROMs. Retrieved may 10, 2010, from Microchip web site: http;/www.microchip.com

[6] Goswani et. al, A. (2009). Design of an Embedded System For Monitoring and Controlling Temperature and Light. International Journal of Electronic Engineering Research; Volume 1 Number $1,27-36$.

[7] Ismaila S. and Momoh M. (2011). Microcontroller based termometer. Energy Soceity Of Nigeria Annual Conference. Sokoto Energy Research Center: Energy soceity of Nigeria.

[8] iWatch. (2009). Wireless temperature seneors and monitoring services to support JCAHO regulatory compliance. Retrieved april 12, 2011, from iwatch TM: http;/iwatch\&flags.com

[9] Kapidere, M. Ahiska, R.and Guler, I. (2005). A New Microcontroller Based Human Brain Hypothermia . System. Journal of Medical Systems Vol29 No5, 27.

[10] Lewis, G. (1995). Patent No. 5386831. United States.

[11] Modem Data Book. (2005). ELG282 Serial Modem data Sheet. Retrieved May 4, 2010, from E-Lines Technology Co.Limited: Htt;//www.e-lins.com

[12] Olavaria, A. A. (2008). Pressure and Temperature Monitoring System For Off-highway Truck Tires. Valparaiso, Chile: Federico Santa Maria Technical University.

[13] Presidio . (2009). improve the health of your network, patients and its investmrnts. Retrieved june 15, 2011, from Presidio Network Solutions web site: http//www.presidio.com

[14] Qingsheng, Z. and Julio, S. (2004) Patent no 7037273 US 
International Journal of Information Sciences and Techniques (IJIST) Vol.3, No.1, January 2013

[15] Raza, A. (2010, Feb 23). Data aquisition and logging system. Retrieved July 4, 2011, from Circuit lake electronics project and circuit collection: www.circuitlake.com

[16] Robert R, Dedrick, John, D. (1999). An Inexpensive, microprocessor-Based Data Logging System. Haley \&Aldrich, New York

[17] Singh, V. (2006). Design and Development of microcontroller Based Temperature and Humidity Controller for Infant Incubator. Patiala, India: Thapar Institute of Engineering Technology.

[18] Smith, S. E. (2010, september 9). What is a Temperature Log? Retrieved june 4, 2011, from wiseGeeK: http//www.getwidget.com

[19] Tiplady, K. (2010, February 2). Remote Critical Area Monitoring System . Retrieved October 4, 2011, from Circuit Lake: http;//www.circuitlake.com 